\title{
Understanding the cognitive restraints of physicians
}

\author{
How Doctors Think \\ (with a new afterword) \\ Jerome Groopman \\ Houghton Mifflin Company, 2008.
}

$\mathrm{T}$ he "physician-turned-popularauthor" is identifiable in many guises. A quick spotter's guide may be helpful: the blockbuster (Michael Crichton); the missionaryadventurer (Wilfred Thomason Grenfell); the medical autobiographer (William Victor Johnston); the doctorstoryteller (Lewis Thomas, Richard Selzer); the medical student exposé (Samuel Shem, Shane Neilson); the literary auteur (William Carlos Williams); the narrator of "believe it or not" patient cases (Oliver Sacks); and the selfhelp/know your disease tour guides (too numerous). Through their writings, these doctors have allowed the public access to the generally closed culture and arcane practice of medicine.

Each in their own way has also offered insights to how doctors think. Recently, a new breed of popular medical author - the critical reasoner has directly addressed this fundamental question. The work of Rhodes Scholar and Harvard surgeon Atul Gawande

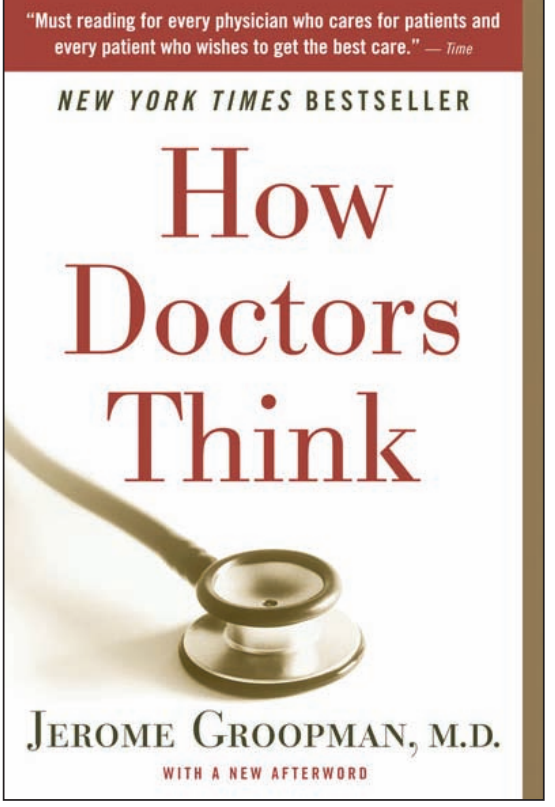

This is a highly successful blend of many of the above mentioned approaches of physician turned author, yet it is different from all of them. While autobiographical, this book is not all about him. And while Groopman exposes some of the problems integral to medicine, he is never alarmist or sensationalist about it. Doctors' stories, patient narratives and explanations of anatomy, physiology, disease and

\section{We need to know how doctors think so that patient safety can be improved.}

(Complications; Better) is one example. The writings of Jerome Groopman, also a professor and doctor at Harvard, fur$\circ$ ther exemplify this recent trend (see www.jeromegroopman.com). Groopman's latest version of his 2007 bestseller, How Doctors Think, now includes an afterword which captures the positive response from patients, colleagues and medical educators to the original work. pathology are recounted here, but all are contextualized within his overarching framework of trying to grapple with how doctors think. Underscoring this book's purpose is the cataloging-inpublication data that categorizes it under decision-making and medical logic.

The heart of How Doctors Think is an analysis of selected medical errors and mistakes, but not those catastrophic events that make news headlines, such as the wrong leg being amputated. Rather, Groopman is after those routine medical cognitive processes that lead some doctors to get locked on to a particular diagnosis and treatment plan and stay with them when both are probably dubious, if not absolutely wrong. He aimed to find out what events, factors or situational causes resulted in the derailing of certain lines of medical reasoning, so that doctors were forced to change their minds to the benefit of the patient.

To facilitate this analysis, Groopman interviewed many colleagues and other notable practitioners as well as patients who had been shuffled through the medical system for years because of what would be revealed as faulty or overly linear medical thinking (one such patient was the author himself). Ultimately, he wanted to write this book so members of the public could critically evaluate the cognitive patterns of doctors in an effort to identify various medical mental traps they might be caught up in.

Physicians wishing to appear to be certain in the uncertain world of medicine, often coupled with their overreliance on technology, account for many of these traps. Chief among these are the "3As" thinking traps: anchoring error — seizing on an initial symptom and making a "snap judgment" that can then trigger a cascade of consequent actions; attribution error - erroneously "stereotyping" patients as certain character types (e.g., alcoholics, complainers or ER "frequent flyers"), which can blind the physician to the existence of an underlying nonbenign condition; and availability error - making a diagnosis on the ease with which it comes to mind because the present patient's case looks very much like other five similar ones the physician saw quite recently. 
These diagnostic booby traps or "heuristics" (translated here by Groopman to mean shortcuts) can be doubly treacherous as they are often grounded in formal medical training, thus they are considered part of normal clinical practice. As such, Groopman also takes a swipe at the trend to rely too much on algorithm or Bayesian-based (statistical) diagnostic thinking and evidence-based medicine. Perhaps, he suggests, it is appropriate to turn the medical aphorism on its tail and to think zebras when you hear hoof beats. This line of reasoning is tantalizing, all the more so as it resonates with that of Kathryn Montgomery's similarly titled How Doctors Think: clinical Judgement and the Practice of Medicine (Oxford UP, 2006), especially the chapter "'Don't Think Zebras': A Theory of Clinical Knowing." We need to know how doctors think so that patient safety can be improved, but also so that medical educators - a group that originally reacted positively to this book - can help realign the training of doctors (under- and postgraduate) to recognize, on the one hand, the utility of the zebra herd mentality, while on the other also understanding its cognitive constraints.

\section{J.T.H. Connor PhD}

John Clinch Professor of Medical

Humanities and History of Medicine Faculty of Medicine

Memorial University

St. John's NL 